\title{
Tick-borne relapsing fever in pregnancy
}

\author{
Haseena Hussein BSc MD, Adrienne Showler MD, Darrell H.S. Tan MD Phd
}

I n August 2012, a previously healthy 34year-old insect ecologist in her 31st week of pregnancy presented to an obstetrics clinic in Toronto, Ontario, with a 2-day history of fever, chills, nausea, vomiting and myalgia. Her symptoms had begun 2 days after returning from a camping trip in southern British Columbia.

She appeared unwell, with mild tachycardia and a maximum temperature of $38.4^{\circ} \mathrm{C}$. Her physical examination was otherwise normal, and the results of initial laboratory investigations were within normal ranges. She was admitted to the obstetrics department with a presumed viral syndrome. An initial infectious workup included blood cultures, serologies for cytomegalovirus, HIV and parvovirus B19, and a nasopharyngeal swab for respiratory viruses.

Forty-eight hours after admission, her fever persisted, her platelet level decreased from $145 \times 10^{9}$ to $27 \times 10^{9} / \mathrm{L}$ (normal $146-429 \times 10^{9} / \mathrm{L}$ ), and her hemoglobin level decreased from 115 to 93 (normal 95-115) g/L. Thrombocytopenia prompted a routine peripheral blood smear, and spirochetes were shown by use of Wright stain (Figure 1).

A more detailed epidemiologic history showed that she had stayed in a farmhouse in Mission, BC, from July 7 to July 9; she had subsequently camped, hiked and swam in fresh water near Lillooet from July 9 to July 12, and had slept in a trailer near Kootenay Lake from July 17 to July 29. She recalled having mosquito bites but no tick bites or rashes. She returned to Ontario on July 31.

Presumed tick-borne relapsing fever was diagnosed, and erythromycin was started. The patient was transferred to the intensive care unit in case of a Jarisch-Herxheimer reaction (Box 1). ${ }^{1}$ Within hours after starting antibiotics, her temperature rose to $38.5^{\circ} \mathrm{C}$, and her heart rate increased to 150 beats $/ \mathrm{min}$. We consulted the hematology service, and after hemolysis, disseminated intravascular coagulation, thrombotic thrombocytopenic purpura and HELLP (hemolysis, elevated liver enzymes, low platelets) syn- drome were ruled out, she was given intravenous immunoglobulin therapy for possible immunemediated thrombocytopenia.

Over the following 24 hours, dyspnea, increased oxygen requirements requiring noninvasive positive pressure ventilation, and bibasilar crackles developed, and pulmonary edema was observed on a chest radiograph. Electrocardiography showed nonspecific ST depression, without a troponin rise. An echocardiogram showed left ventricular hypokinesis with an ejection fraction of $45 \%$ (normal $55 \%-70 \%$ ) and a right ventricular systolic pressure of 40 (normal 1530) $\mathrm{mm} \mathrm{Hg}$. Severe thrombocytopenia persisted despite the patient receiving 1 unit of pooled platelets and 2 doses of immunoglobulin administered intravenously. Her medication was then changed to intravenously administered ceftriaxone for possible myocarditis related to tick-borne relapsing fever and in case of co-infection with leptospirosis, given her exposure to fresh water. Doxycycline was added after a discussion of the risks and benefits of its use in pregnancy with the patient. Two doses of betamethasone were administered because of the risk of preterm birth, and furosemide was administered for diuresis.

By the eighth day in hospital, major improvement in the patient's hypoxia, fever, platelet count and overall clinical status were noted. Doxycy-

\section{- Ker pOINTS}

- In Canada, tick-borne relapsing fever is an uncommon infectious disease endemic in southern British Columbia, characterized by episodic fever, headache and myalgia.

- Thrombocytopenia commonly occurs in patients with tick-borne relapsing fever, and it may be severe.

- The diagnosis can usually be made by detecting spirochetemia on a peripheral blood smear during acute febrile episodes, and it can be confirmed in reference laboratories using immunofluorescent stains and polymerase chain reaction.

- Patients should be monitored for the Jarisch-Herxheimer reaction following treatment, and they should be provided with supportive care as needed. 
cline was stopped after 4 doses, and erythromycin with ceftriaxone were resumed. Serology for Epstein-Barr virus, HIV, West Nile virus, Borrelia burgdorferi, Rocky Mountain spotted fever and murine typhus were negative. Polymerase chain reaction (PCR) testing performed at the National Microbiology Laboratory indicated that her blood contained Borrelia hermsii. The patient later discovered that a squirrel's nest had been recently removed from the trailer at Kootenay Lake; this was the likely source of exposure. She was discharged home on the 12th day in hospital and completed 14 days of erythromycin. Repeat echocardiography performed 1 month after discharge was normal, and she later gave birth to a healthy baby girl at term. Histopathologic examination of the placenta revealed no evidence of spirochetes, chorioamnionitis or villitis.

\section{Discussion}

Tick-borne relapsing fever is caused by spirochetes of the Borrelia genus, most commonly B. hermsii in North America. Ornithodoros "soft" ticks transmit $B$. hermsii through painless nocturnal bites that last less than 30 minutes; thus, most patients with tick-borne relapsing fever are unaware that they were exposed. Ornithodoros ticks live near the nests of their animal reservoirs, which include mice, chipmunks, squirrels and other small mammals. ${ }^{1}$ Humans may become infected while sleeping in rodentinfested dwellings, typically at altitudes of 450 $2450 \mathrm{~m}$ and usually during the summer. ${ }^{2}$

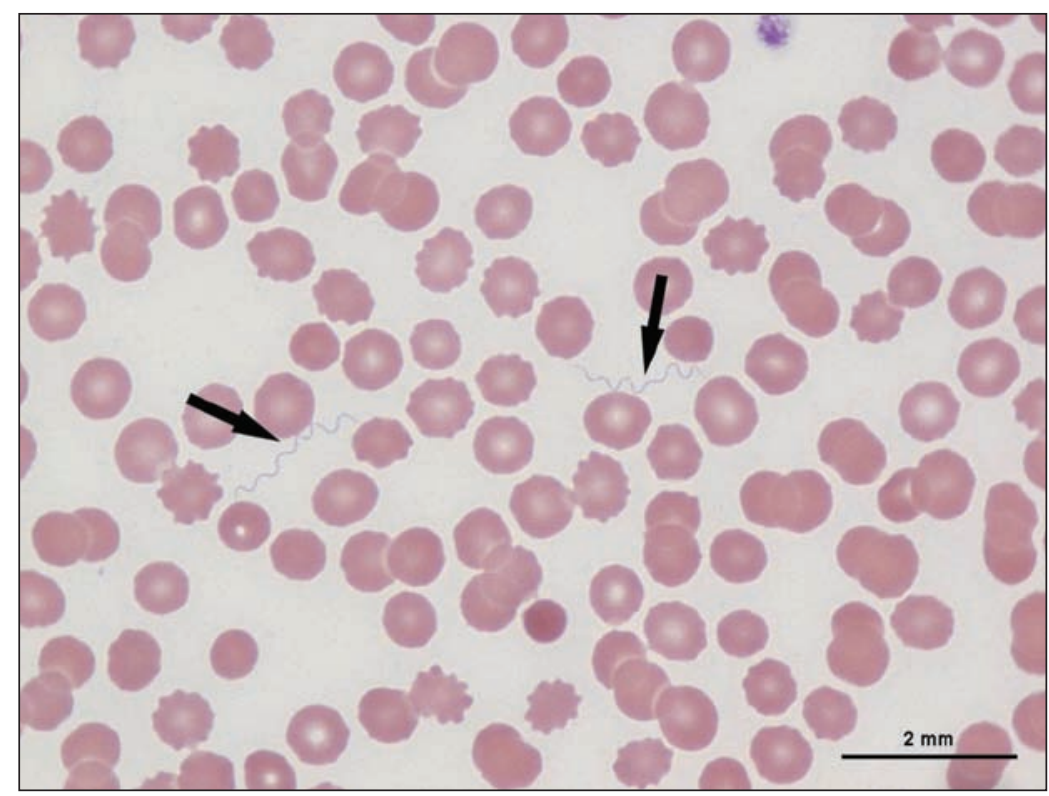

Figure 1: Wright-stained peripheral blood smear from a pregnant 34-year-old woman with fever, myalgias and vomiting after camping in British Columbia. Spirochetes compatible with tick-borne relapsing fever are visible (arrows) (original magnification $\times 100$ ).

\section{Box 1: Jarisch-Herxheimer reaction}

Jarisch-Herxheimer reaction is an inflammatory reaction characterized by fever, tachycardia and hypotension. It frequently occurs within hours of starting antibiotic treatment for tick-borne relapsing fever, syphilis and other spirochete infections. The reaction is linked to cytokine release as spirochetes are cleared from the circulation.

In North America, tick-borne relapsing fever is endemic in the western United States and southern BC, primarily in the Okanagan Valley and West Kootenay area. ${ }^{2.3}$ Although only about 50 cases of tick-borne relapsing fever have been documented in the $\mathrm{BC}$ interior in the past 20 years (Muhammad Morshed, BC Public Health Microbiology and Reference Laboratory: personal communication, 2012), this is likely an underestimate, because $B$. hermsii infection is often underrecognized and is not a reportable disease in Canada. Other Borrelia species that cause tick-borne relapsing fever are endemic in parts of Mexico, Central and South America, east and southern Africa, the Mediterranean region and the Middle East.

After a 2-18 day incubation period, patients with tick-borne relapsing fever experience fever associated with nausea, headache and myalgia. Fevers last a median of 3 days and are followed by an afebrile period that lasts $4-14$ days. ${ }^{1}$ Febrile paroxysms may recur for up to 13 episodes. Thrombocytopenia commonly accompanies acute disease. Hemorrhage frequently occurs in patients with the closely related louseborne relapsing fever, but major bleeding complications are uncommon in tick-borne relapsing fever, even among patients with platelet counts below $20 \times 10^{9} / \mathrm{L}^{2}$ Prolonged QTc interval and ST changes can occur with myocardial involvement, although congestive heart failure from tick-borne relapsing fever is unusual. ${ }^{4}$ In our patient's case, it is unclear whether her congestive heart failure, low ejection fraction and ST changes were related to direct effects of $B$. hermsii or to intravenous immunoglobulin administration, iatrogenic volume overload or myocardial depression in the context of acute systemic illness and Jarisch-Herxheimer reaction. Other less common manifestations of tick-borne relapsing fever include jaundice, hepatosplenomegaly, confusion and central nervous system involvement with nuchal rigidity and meningitis.

Tick-borne relapsing fever is usually diagnosed by light-field microscopic examination of WrightGiemsa-stained peripheral blood smears. During acute febrile episodes, only $70 \%$ of patients will have visible extracellular spirochetes on a periph- 
eral blood smear; ${ }^{5}$ for detection, the density of spirochetemia is in the range of $10^{4}-10^{5}$ per $\mathrm{mL}$ blood. ${ }^{1,3}$ Although several other arthropod-borne and spirochetal infections that cause acute febrile illness occur in Canada, the Borrelia species implicated in tick-borne relapsing fever and louseborne relapsing fever are the only spirochetes that can be visualized by light microscopy (Appendix 1, available at www.cmaj.ca/lookup/suppl/doi:10 .1503/cmaj.122053/-/DC1). Immunofluorescent staining, dark-field microscopy and serologic testing can also be performed. A false positive result of an enzyme-linked immunosorbent assay (ELISA) or indirect immunofluorescent antibody test (IFA) can occur because of cross-reactivity with other bacteria, including $B$. burgdorferi, the spirochete that causes Lyme disease. ${ }^{2}$ Immunoblot testing is recommended for samples that test positive via ELISA or IFA to characterize the pattern of reactivity; this may identify false positives. Although not commercially available, serologic assays using the recombinant protein GlpQ can also distinguish between tick-borne relapsing fever and false positives due to other spirochetes. Conversely, B. burgdorferi ELISA and occasionally Western blot results may be positive for patients with high $B$. hermsii antibody titres, leading to a potential misdiagnosis of Lyme disease in areas where these diseases are both endemic. ${ }^{2}$ Confirmatory PCR testing for $B$. hermsii is available only at reference laboratories in Canada. In our case, the diagnosis was made serendipitously by manual examination of a blood smear performed for detection of thrombocytopenia; the diagnosis was later confirmed by PCR at the National Microbiology Laboratory.

Tetracyclines are first-line treatment for acute tick-borne relapsing fever, although penicillin and erythromycin are also effective. ${ }^{1,2}$ Evidence supporting the use of tetracyclines over alternate regimens is limited to in vitro data, animal studies and expert opinion. ${ }^{6,7}$ Ceftriaxone remains the drug of choice for involvement of the central nervous system and is recommended for other forms of Borrelia infection with myocardial involvement. ${ }^{8} \mathrm{~A}$ Jarisch-Herxheimer reaction, characterized by fever, tachycardia and hypotension, occurs within hours of antibiotic treatment in up to $50 \%$ of cases, ${ }^{1}$ as in our patient's case. Intravenous im munoglobulin is not known to be beneficial in thrombocytopenia related to tick-borne relapsing fever. In animal studies, B. hermsii caused thrombocytopenia through direct platelet binding and subsequent platelet clearance, rather than via immune-mediated mechanisms. ${ }^{9}$ Early empiric treatment with antibiotics should be considered in the appropriate clinical setting, although most patients spontaneously recover from tick-borne relapsing fever without treatment. Death due to tick-borne relapsing fever is rare in North America.

\section{Risk during pregnancy}

Only 12 cases of tick-borne relapsing fever during pregnancy have been reported in North America. ${ }^{5}$ Major maternal complications can occur, including acute respiratory distress syndrome, renal and splenic infarcts, and severe Jarisch-Herxheimer reaction. ${ }^{5,10}$ Studies from rural East Africa have shown an increased incidence of spontaneous abortion, low neonatal birth weight and preterm labour among pregnant women with tick-borne relapsing fever. ${ }^{6,11}$ Although women infected during pregnancy had higher levels of spirochetemia, mortality rates of about $1.5 \%$ were observed in both pregnant women and age-matched controls with tickborne relapsing fever. ${ }^{11}$

Thrombocytopenia typical of acute tick-borne relapsing fever poses an increased risk of hemorrhage in both mother and baby around the perinatal period. Transplacental transfer of B. hermsii or acquisition during delivery may result in neonatal infection. Of the 11 neonates born to B. hermsii-infected mothers in North America, 6 acquired perinatal tick-borne relapsing fever, resulting in 2 infant deaths: 1 because of meningitis associated with splenic abscesses, and 1 because of high-level spirochetemia complicated by liver abscess. ${ }^{1,5}$

Evidence to inform treatment decisions during pregnancy is limited. Tetracycline and doxycycline are labelled as category D medications in pregnancy, and they are therefore recommended only in the absence of effective alternative treatments. Fetal exposure to tetracycline may result in permanent yellowish-brown tooth discoloration and reversible delayed skeletal development. ${ }^{12}$ Although these adverse events have not been documented with fetal doxycycline exposure, they cannot be ruled out, given the limited data on its use in pregnancy and the possibility of a tetracycline class effect. Tetracyclines are considered first-line treatment for tick-borne relapsing fever in nonpregnant patients; however, this recommendation is primarily based on in vitro data, animal studies and expert opinion. ${ }^{6,7}$ Because no convincing evidence has shown the superiority of tetracyclines over other agents, it is recommended that pregnant women be given penicillin or erythromycin. ${ }^{13}$ Treatment failures in pregnancy have, however, been documented with both agents, perhaps because of the persistence of spirochetes in the placenta. ${ }^{11,14}$ Patients should be closely monitored for treatment failure both after completing antibiotic therapy and around the time of delivery. 


\section{Conclusion}

In our patient, the initial impression was of a nonspecific viral illness, and we focused on ruling out infections of particular clinical relevance in pregnancy, including cytomegalovirus, HIV and parvovirus. The diagnosis was ultimately established based on the unexpected finding of spirochetes on a peripheral blood smear performed to investigate thrombocytopenia. This case illustrates the importance of a detailed travel history, which should include both travel abroad and within Canada.

There is substantial geographic variation in infectious disease epidemiology throughout Canada, and this case also highlights the need for increased physician awareness of endemic infections in other provinces. Tick-borne relapsing fever is currently a reportable disease in 12 western US states, ${ }^{5}$ a policy that should be considered in Canada because this disease meets many of the criteria for reportable disease status articulated by the Public Health Agency of Canada. ${ }^{15}$ Ongoing disease surveillance is particularly important because new endemic areas may emerge with climate change. Projections based on mathematical modelling predict a shift toward higher altitudes, because soft ticks depend on microclimates with limited temperature variation and stable humidity. ${ }^{16}$

Reportable disease status for tick-borne relapsing fever would improve epidemiologic monitoring and facilitate early diagnosis through heightened physician awareness. Measures leading to prompt recognition and treatment may be life-saving in immunocompromised hosts, who have the potential for severe complications. As this case illustrates, although pregnant patients are at risk for more severe disease, both mother and child can have good outcomes with early diagnosis, close monitoring and prompt antibiotic treatment.

\section{References}

1. Dworkin MS, Schwan TG, Anderson DE Jr. Tick-borne relapsing fever in North America. Med Clin North Am 2002;86:41733. viii-ix.

2. Dworkin MS, Anderson DE Jr, Schwan TG, et al. Tick-borne relapsing fever in the northwestern United States and southwestern Canada. Clin Infect Dis 1998;26:122-31.

3. Banerjee SN, Banerjee M, Fernando K, et al. Tick-borne relapsing fever in British Columbia, Canada: first isolation of Borrelia hermsii. J Clin Microbiol 1998;36:3505-8.
4. Wengrower D, Knobler H, Gillis S, et al. Myocarditis in tickborne relapsing fever. J Infect Dis 1984;149:1033.

5. Tick-borne relapsing fever in a mother and newborn child. MMWR Morb Mortal Wkly Rep 2012;61:174-6.

6. Goubau PF, Munyangeyo C. Tick-borne relapsing fever and pregnancy. A clinical study in Rwanda. Ann Soc Belg Med Trop 1983; 63:347-55.

7. Southern PM Jr, Sanford JP. Relapsing fever: a clinical and microbiological review. Medicine 1969;48:129-49.

8. Tick-borne relapsing fever: information for clinicians. Atlanta (GA): Center for Disease Control and Prevention; 2012. Available: www.cdc.gov/relapsing-fever/clinicians/ (accessed 2012 Dec. 7).

9. Alugupalli KR, Michelson AD, Joris I, et al. Spirochete-platelet attachment and thrombocytopenia in murine relapsing fever borreliosis. Blood 2003;102:2843-50.

10. Davis RD, Burke JP, Wright LJ. Relapsing fever associated with ARDS in a parturient woman: a case report and review of the literature. Chest 1992;102:630-2.

11. Jongen VH, van Roosmalen J, Tiems J, et al. Tick-borne relapsing fever and pregnancy outcome in rural Tanzania. Acta Obstet Gynecol Scand 1997;76:834-8.

12. Nahum GG, Uhl K, Kennedy L. Antibiotic use in pregnancy and lactation. Obstet Gynecol 2006;107:1120-38.

13. Dworkin MS, Schwan TG, Anderson DE, et al. Tick-borne relapsing fever. Infect Dis Clin North Am 2008;22:449-68.

14. Horton JM, Blaser MJ. The spectrum of relapsing fever in the Rocky Mountains. Arch Intern Med 1985;145:871-5.

15. Final report and recommendations from the National Notifiable Diseases Working Group. Can Dis Wkly Rep 2006;32:211-25. Available: www.phac-aspc.gc.ca/publicat/ccdr-rmtc (accessed 2013 Apr. 26).

16. Johnson TL. The ecology of tick-borne relapsing fever in Western North America [dissertation]. Missoula (MT): The University of Montana; 2012.

Affiliations: Department of Medicine (Hussein, Showler, Tan); Division of Infectious Diseases (Showler, Tan), University of Toronto; Division of Infectious Diseases (Tan), St. Michael's Hospital, Toronto, Ont.

Contributors: All of the authors contributed substantially to writing the manuscript. Adrienne Showler and Haseena Hussein wrote the original draft of the manuscript, which was revised by Darrell Tan. All of the authors approved the final version submitted for publication.

Acknowledgement: The authors thank Dr. Hasan Ghaffar from the St. Michael's Hospital Department of Pathology for providing the blood film image.

The section Cases presents brief case reports that convey clear, practical lessons. Preference is given to common presentations of important rare conditions, and important unusual presentations of common problems. Articles start with a case presentation (500 words maximum), and a discussion of the underlying condition follows (1000 words maximum). Visual elements (e.g., tables of the differential diagnosis, clinical features or diagnostic approach) are encouraged. Written consent from patients for publication of their story is a necessity and should accompany submissions. See information for authors at www.cmaj.ca. 